\title{
The ethical and legal aspects of image publications of patients: an integrative literature review
}

\begin{abstract}
Advances in information technology have made it possible to be in line with health and ensure the safety of patient data, since capture to sharing. However, there are still issues that need to discuss, especially in the area of health, regarding patient autonomy, in its biopsychosocial and spiritual perspective. Thus, this study aimed to highlight and discuss the ethical and legal aspects of the publication of patient images, based on scientific health publications. It is an Integrative Review of Literature, which aims to summarize the data present in the empirical and theoretical literature to broaden the understanding of a particular phenomenon. Performed the search in the Virtual Health Library (BVS) and from the study criteria, six articles were included. The final sample of this review observed a few original studies on the ethical and legal aspects of the use of patient images by health professionals, published by researchers from the United States of America, France, England, Australia, Brazil, and Colombia. The results of this review evidenced the fragility regarding the use of the consent term and the use and sharing of images, with the perspective of preserving the autonomy of the patient. The study presented the need for ethical and legal devices, that reveal greater attention and surveillance with the publication of patient images by students and health professionals and suggests the use of protocols that aim at storing the images, avoiding unnecessary sharing.
\end{abstract}

Keywords: ethics, legislation, confidentiality, biomedical technology, information technology
Volume 9 Issue $6-2018$

\author{
Shirley Maria de Araújo-Passos, Waldeyde \\ Magalhães dos Santos, George Lucas
} Augusto Trindade da Silva, Isabela Cristina de Miranda Gonçalves

Research and Development Center UNA-SUS Amazônia,

University of the State of Amazonas, Brazil

Correspondence: Shirley Maria de Araújo-Passos, Research and Development Center UNA-SUS Amazônia, University of the State of Amazonas, Av. Carvalho Leal, 1777 - Cachoeirinha, Manaus - AM, CEP: 69065-00I, Brazil, Tel +55 (92) 9 9I52-24I0, Emailshirley.passos@unasusamazonia.com.br

Received: December 12, 2018 | Published: December 28 2018

\section{Introduction}

The United Nations (UN), through the Universal Declaration on Bioethics and Human Rights, establishes that the subject submitted to any medical intervention, must inform before carrying out any procedure, when unable to consent, this task belongs to the legal guardian. It is also highlight that patient can remove the consent at any time, without prejudice or prejudiced imposed about him. ${ }^{1}$ Advances in information technology have made it possible to be in line with health and ensure the safety of patient data from capture to sharing. However, there are still issues that need to be respected when it comes to the health area, since it is necessary to consider the autonomy of the patient, in its biopsycho spiritual perspective. In this context, the 1997 Convention on Human Rights and Biomedicine provides for the consent of the subject for any action that refers to the manipulation of elements inherent to the patient integrity. Therefore, in order to capture patient pictures, it is necessary for the patient to grant free and informed consent. ${ }^{2}$ The technological advent revealed new forms of communication among health professionals. Accordingly, discussions on bioethical barriers started in the academic and care area, in order to find better measures that would protect the bioethical integrity of professionals, institutions, and patients. In the case of data protection, Law No. 7,232 of October 29, 1984, establishes in item VIII of art. 2 , the need for "legal and technical mechanisms and instruments for the protection of the confidentiality of stored data, processed and transmitted, of the interest of privacy and security of natural and legal, private and public person". ${ }^{3}$

The Brazilian civil code explicitly states in article 5 that privacy, private life, honor, and image are inviolable and that transgression of these rights may reveal compensation for moral or material damages. The same text establishes in article 20 that only if authorized, or if legally requested, the publication, exhibition or use of the image of a person may be prevented by request of the subject, without any prejudice to the applicant. Moreover, if the individual is dead or absent, the spouse, ascendant or descendant may require that right. ${ }^{4}$ The use of patient data in research in Brazil is regulated by the National Health Council, which establishes that the patient should have their autonomy and privacy preserved, for this he must sign the Written Informed Consent Form (WICF) to support both the subject of the research, as well as the researcher of any bioethical obstacle. In the case of persons incapable of consent, such as children, it is up to the parents, legal guardians or legal representatives to sign the WICF and the subject confirm their participation in the research through the Consent Form Free and Informed. In this way, the researcher can convey images of the patient for scientific purposes, as expressed in the term. ${ }^{5,6}$ In this sense, the objective was to highlight and discuss the ethical and legal aspects of the publication of patient images, from publications health sciences.

\section{Methods}

In order to carry out this study, used the integrative review, a specific method, which aims to summarize the data present in the empirical and theoretical literature to extend the understanding of a particular phenomenon. Thus, having been fulfilled six established stages:

1. Identification of the problem

2. Literature and article search

3. Evaluation of the articles in pairs

4. Analysis of articles included for review

5. Evaluation and interpretation of results

6. Presentation and discussion of the review. 
Although systematic review and meta-analysis are the most important method of review, they do not include important descriptive questions about a situation. ${ }^{7}$ To guide the integrative review, the following guiding question was formulated: What are the ethical and legal aspects that are interrelated with the registration, publication and sharing of images of patients among health professionals? In order to select the articles, performed the search in Virtual Health Library (VHL). The review performed based on the Prisma methodology - Preferred Reporting Items for Systematic Reviews and MetaAnalyzes (Figure 1).

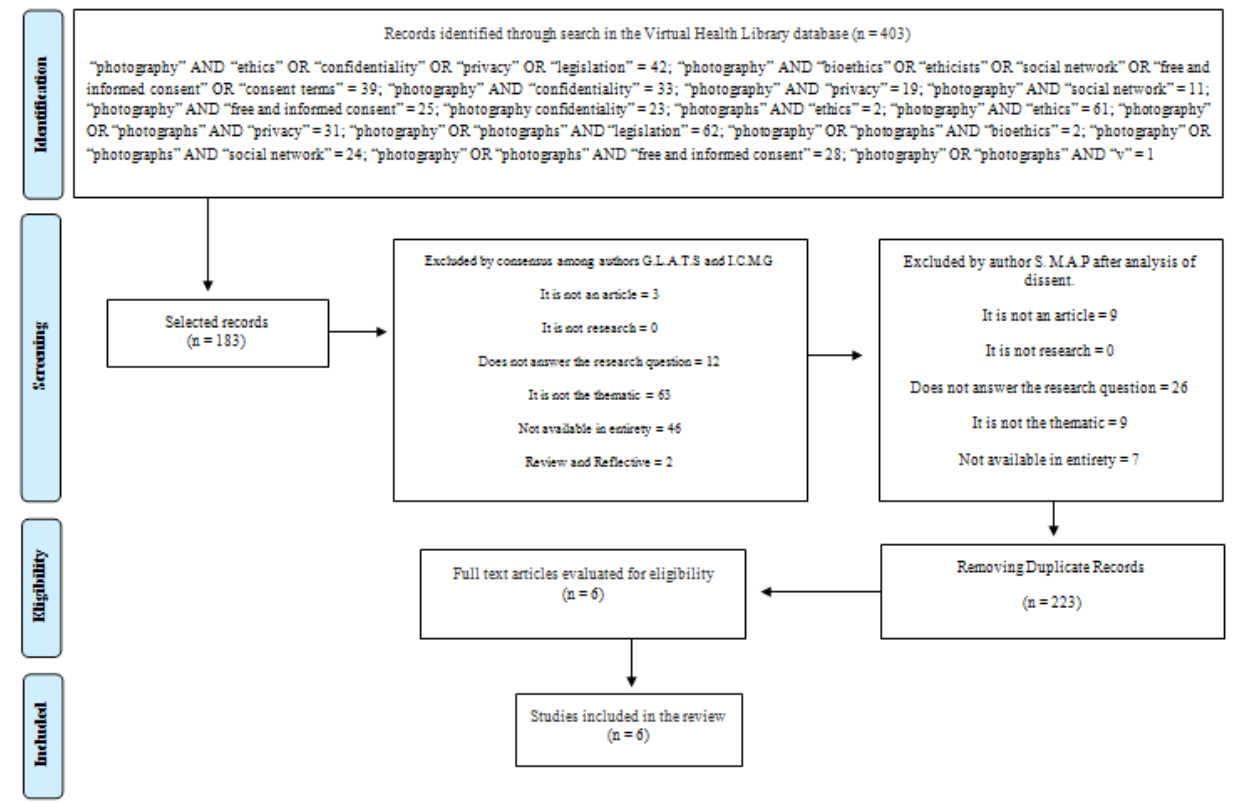

The Health Sciences Descriptors (DeCS) used were: photography; ethics; legislation; terms of consent; privacy; social network; bioethics; confidentiality. These elements were combined using the Boolean operators "AND", "OR" and "AND NOT", always having as main descriptor the word "photography". The criteria for inclusion of the articles defined for this integrative review were:

1. Original research articles.

2. Published in Portuguese, English, and Spanish.

3. Between the years 2013 and 2017.

4. Available in full free of charge.

5. To respond to the research topic.

The excluded were articles of review, critical reflection, those that did not deal with ethical and legal aspects for the use of patient images, and publications in the form of theses and dissertations. ${ }^{8}$ The selection of the articles accomplished in July and August 2018, following the inclusion and exclusion criteria. After the selection of the articles, they were evaluated in the content of the title and in the abstract by two independent researchers from the criteria defined to arrive at the consensus. A third evaluator read the divergent studies to define the articles that would read in its entirety read. Realized the articles predefinition for complete reading and categorization by each reviewer. Subsequently, effected a comparison between the researchers about the content and attendance to the proposed theme (Figure 1).

\section{Results}

The final sample of this review observed a few original studies on the ethical and legal aspects of the use of patient images by health professionals, published by researchers from the United
States of America, France, England, Australia, Brazil, and Colombia respectively, as presented in Table 1, in addition to the specifications of each article.

From the critical reading of the articles were identified two central themes: Informed Consent and Use, and storage of patient images. Two articles discuss informed consent for photographs and four articles discuss the use and storage of patient images. ${ }^{9-14}$

Regarding the first thematic, which informs consent for photographs, cited two types of consent: verbal and written. In the Australian research, verbal consent was the most common one, justified by doctors and nurses who are more concerned with everyday practice. Consent to take clinical photographs should be informed and doctors have a legal requirement to use the photograph as defined by that consent, so this should include an explanation of the purpose for which a photograph will be used and for whom the photograph will likely be shown. ${ }^{11}$ Already in the French study reported the use of clinical images of patients by health professionals as a way to broaden the scope of the multidisciplinary team meetings, exemplifying the possibility of monitoring the evolution of the lesion by photography for a better decision. ${ }^{10}$ For Australian researchers discuss the problems arising from the sharing of photographs of patients in the public domain by health professionals, which may lead to legal problems for the hospital, its patients and employees. The authors address the lack of professional knowledge and non-compliance with cell phone policies and usage..$^{11}$ Two articles mention the publication and dissemination of photographs of patients during medical care and dental procedures on sites and personal profiles, as before and after. ${ }^{12,13}$ They argue that publications and patient photographs sharing without regard to ethical implications may entail ethical and legal implications for these professionals. 
Table I Characterization of the scientific production about the use of patient images in the practice of health professionals

\begin{tabular}{|c|c|c|c|c|}
\hline Origin & Title & Authors & $\begin{array}{l}\text { Journal (edition, } \\
\text { number, page, } \\
\text { year) }\end{array}$ & Purpose of the study \\
\hline USA & $\begin{array}{l}\text { Sharing clinical photographs: } \\
\text { Patient rights, professional } \\
\text { ethics, and institutional } \\
\text { responsibilities }\end{array}$ & $\begin{array}{l}\text { Means, Jacquelyn } \\
\text { M; Kodner, Ira J; } \\
\text { Brown, Douglas; Ray, } \\
\text { Shuddhadeb. }\end{array}$ & $\begin{array}{l}\text { Bull Am Coll Surg; } \\
\text { 100(10): } 17-22 \text {, } \\
2015 \text { Oct. }\end{array}$ & $\begin{array}{l}\text { Describe a common scenario in which a resident is seeking } \\
\text { the advice of a surgeon on duty to consult a patient case, } \\
\text { and asked to share a photograph of the patient's injury. } \\
\text { Possible approaches to this dilemma are described, as well } \\
\text { as ethical issues that need to be considered in choosing } \\
\text { one option over another. }\end{array}$ \\
\hline
\end{tabular}

\section{Interest of including trauma photography in the picture archiving and communication system of a teaching hospital}

Taking ethical photos of children for medical and research

England purposes in low-resource settings: an exploratory qualitative study policy, ethics and practice in an Australian tertiary hospital.

Analysis of the profiles of dental clinics and surgeons-dentists in two social networks as far as the ethical, propaganda and advertising aspects

Management of electronic social networks by medical students:

Colombia the case of the publication of photographs of patients and the medical offerings
Bronsard, N; Chignon Sicard, B; Amoretti, N Rottier, H; Ertz, P; de Peretti, F.

\section{Devakumar, Delan;} Brotherton, Helen; Halbert, Jay; Clarke, Andrew; Prost, Audrey; Hall, Jennifer.

Burns, Kara; Belton, Suzanne.

Arthur Igor Cruz Lima, Rafael Andrade Cruz, Ricardo Araújo da Silva

Pedro José Villamizar, Sandra Milena Moreno, Freddy Moreno
Orthop Traumatol Surg Res; 101(3): 387-90, 2015 May.

BMC Med Ethics; 14: 27, 2013 Jul 09

Aust Health Rev; 37(4): 437-41, 2013 Sep.

RBOL 2016;3(2):66-73 36:140-8
To describe a procedure for the inclusion of photographs taken by the various agents involved in traumatology in a teaching hospital's archiving and communication system (PACS) in a reliable, reproducible and confidential way. xplore issues around photography in resource-poor places, focusing in particular on the challenges of obtaining informed consent.

Clarify the possibility of widespread clinical medical photography in a tertiary hospital in Australia, examining the legal and ethical implications of this practice.

Evaluate two social networks (Facebook ${ }^{\circledR}$ and Instagram ${ }^{\circledR}$ ) of dental surgeons and dental clinics in compliance with the provisions of the Code of Dental Ethics (CEO), as well as verify ethical infractions committed in these social networks.

To determine the management of electronic social networks by medical students, regarding the publication of photographs with patients.

\section{Discussion}

It was presented a discussion for the professional decision between verbal informed consent versus written informed consent, in the United States, in which verbal consent would certainly be faster, easier and less intrusive, being this approach defender to the ethical principle of non-maleficence in order to minimize suffering. However, from a legal point of view, written consent may be the recommended option to protect the doctor and hospital from future liabilities. Recommended that health institutions have existing standards and policies to address this problem, and institutions should facilitate this process of making policies available and compatible with the use of technologies. ${ }^{9}$

Three articles presented the legal aspect of the use of photography. ${ }^{10,13,14}$ In the French context, according to the Public Health Code individual consent is not required when the use of the image is for the purpose of saving lives, even if the patient is identified. ${ }^{10}$ In the United Kingdom, the General Medical Council (GMC), in March 2013, established guidelines for the making and use of patient images and audio, emphasizing the need for informed consent for visual and audio recordings, including photographs. ${ }^{14}$ The third article dealing with the legal aspect of the use of photography in the context of Colombia, the discussion is based on patients' rights to their medical records, which is private and should only be known to third parties, depending on their authorization. It is

determined that the patient's health records should be recorded on the patient's health, medical acts and other procedures, chronologically by the health team. Thus, photographs taken from patients, even if they have been authorized, are part of the clinical history and legal, technical, scientific and administrative documents pertinent to the care processes. ${ }^{13}$ In England, the article presents the patients' point of view, in which the overwhelming message was the need for consent when taking photographs of children. Furthermore, they suggest the importance of considering photographic images with the same degree of care and rigor that apply to other forms of data and acting appropriately to protect its storage and use. According to this article, the barriers to obtaining consent were listed as being: the difference in the concept of informed consent; language; understanding; type of consent, and power disparity in the relationship. ${ }^{14}$ There has been an agreement that consent is needed, but exist varying views on how to obtain it, by whom, from whom and when. Considered the obtaining full informed consent as the greatest challenge to the ethical dissemination of clinical images.

In the United States, the article describes an ethical dilemma in modern surgical practice and alerts physicians and residents to sharing images through mobile devices in hospitals and medical institutions. The article proposes that those responsible for these images have a greater concern and knowledge "that taking a picture of a patient for clinical and educational purposes may present ethical problems". 9 
Hospitals and medical institutions need to maintain confidentiality and safeguard patient images, so the professionals must know the fragility in the sharing of images by mobile devices and establish measures for image security in order to protect not only the patient but also the health professional and the institution itself. It is necessary for the patient to know the destination of the clinical images, because with the greater advancement of technology and greater connectivity, increase the chances of sharing of a photograph or digital clinical image improperly, thus hurting the privacy and confidentiality rights of the patient. Informed consent should be the means by which all uses of patient imaging should be clarified, both in the medical record and in the follow-up of treatment, in teaching presentations and in scientific publications. ${ }^{9}$

In Brazil, the National Health Council regulates the use of personal data in health research, a consent must be requested for any photograph that exposes any part of the patient, since this is not an anatomical piece, but an individual with specifics and conceptions. ${ }^{5}$ In recent years, there has been a significant increase in the use of information technology as a complementary tool in the health area, for example, implementation of electronic medical records, medical consultations using telehealth and telemedicine, and the use of mobile electronic devices and smartphones for capturing images. All these technologies bring a fragility regarding the legal and ethical aspects considered in the use of photographs for patient management, health education and research in medical practice. The French article described the ways of storing patient trauma photographs within the Picture Archiving and Communication System (PACS), an institutional software of interaction between the photographs, in Nice in Italy. The system allows the images of trauma captured by physicians to be safely shared in an image archive, and only those involved with the patient's treatment will be able to access the images of the traumas safely, allowing them to be consulted in several stages, both by doctors, medical specialists, or by even the hospital for defense in legal proceedings. Medical records in computerized systems can bring greater benefits to patients' treatments, with lower costs for the institution.

In Australia, a study was conducted on the possibility of using clinical photography in a tertiary hospital, examining the legal and ethical implications of this practice. The research found that $48 \%$ of the doctors interviewed took clinical photographs using hospitalowned cameras. They detected several errors in the use and in the patient's photographs' use, in the way that professionals captured the patients' photographs, the use of the images by the professional team, and the type of informed consent of the patients. Being the most worrying that a significant number of clinicians performed storing of images on personal mobile devices, risking security of patient information. The research encourages the hospital to develop a plan of action in the use of clinical photography in order to prevent the distribution of patient data to the public domain. ${ }^{11}$ In Brazil, a research was identified that evaluated two social networks (Facebook ${ }^{\circledR}$ and Instagram ${ }^{\circledR}$ ) of dental surgeons and dental clinics in compliance with the provisions of the Code of Dental Ethics (CDE). Used a questionnaire based on the ethical infractions quoted in the CDE and considered photographs and legends in the studied social networks, a total of 257 pages, were verified ethical infractions committed in these social networks. $97.28 \%$ of the social networks surveyed were at odds with at least one of the 14 items investigated. The authors suggest a greater control of these social networks, aiming respect to the users of the services, as well as updating of the researched professionals, with the precepts of the Code of Ethical Dentistry in force. ${ }^{12}$ In Colombia, was conducted a study that characterized the medical students of Pontificia Universidad Javeriana de Cali, in order to determine the management of electronic social networks by medical students, referring to the publication of photographs with patients. $97,6 \%$ of the students surveyed stated that they were active users of at least one of the main electronic social networks and $17.6 \%$ said they had published at least one photograph taken during patient care. It was found in the survey that the vast majority of students had smartphones and had published their user profile on major electronic social networks, through which they released photographs of patients during medical care, which could have ethical and legal implications, going against medical professionalism. ${ }^{13}$ In Brazil, the Code of Medical Ethics in Chapter VI, which deals with human rights, establishes as prohibiting medical professionals, in its Art. 24 "Failure to guarantee to the patient the exercise of the right to decide freely on his or her person or his or her well-being, as well as exert his or her authority to limit it". Therefore, to publish a photograph taken during the care or treatment to the patient in social networks is prohibited to the medical professional in Brazil, except with the consent and authorization of the patient. ${ }^{15}$

\section{Conclusion}

This integrative review presented the concern that the authors of the selected articles had in relation to the use and sharing of images, both for follow-up of patient's treatment and for advertising in social networks. Most health professionals are either unaware of the ethical precepts of sharing images or do not care about patient autonomy regarding the use of their image, not respecting their privacy and confidentiality. In consultation with the Brazilian Federal Council of Medicine (FCM), the public body said that the consent form (CT) signed by the patient is not necessary if the photograph or sequence of photographs for follow-up of the lesion does not allow identification of the patient. However, if published this same image on the internet for the purpose of research and/or education of health professionals, it is necessary to sign the CT. The dissemination of images by medical students is the responsibility of the preceptor and of the academic. ${ }^{16}$ The Code of Ethics Dentistry establishes as an infraction, in its Article 14, "III - to refer to identifiable clinical cases, patient display, its image or any other element that identifies it, in any medium of communication or under any pretext". In Brazil, dentistry professionals use social networks to help them search and maintain clients, through advertisements and case shows. Just can exhibit the patient's images if "the dental surgeon is in the exercise of teaching or in scientific publications, where the patient's authorization or legal guardian allows him to expose the image $[. .]. "{ }^{17}$ Thus, patient images may be exhibited for scientific purposes or in the exercise of teaching, and with the consent of the patient. Resolution No. 554/2017 of the Brazilian Federal Nursing Council (COFEN) establishes the guiding criteria for practices of use and behavior of nursing professionals in the mass media: in the print media, in advertisements, in urban furnishings and social media. Article 4 of the aforementioned resolution emphasizes that not allows the nursing professional to "expose the image of patients in social networks and social groups such as WhatsApp" as well as "expose images of the face or body of patients that are not intended for academic purposes". ${ }^{18}$ It is believed that even professional advice waiving, in certain cases, the signature of the term, patients need to have their autonomy to decide on the registration, use and sharing of their image, knowing where and when the captured image will be used. The Term of Commitment 
is the document that could provide the legal support for both the health professional and the institution to which it is linked. It is also important to highlight the need to advance in the regulation for the storage of images of patients used in clinical treatment, teaching, and research in hospitals and universities. It is necessary to use protocols that aim these storages safely and effectively, avoiding unnecessary sharing.

\section{Funding details}

Samsung Electronics of the Amazon LTDA by means of the Amazon Informatic Law, it foments research and technological development of the region in partnership with the University of the State of Amazonas.

\section{Acknowledgments}

We thank Samsung Electronics of the Amazon LTDA by means of the Amazon Informatic Law; it foments research and technological development of the region in partnership with the University of the State of Amazonas, and the Research and Development Center UNASUS Amazonia of the said university.

\section{Conflicts of interests}

Authors declare that there is no conflict of interest.

\section{References}

1. United Nations Organization. Universal Declaration on Bioethics and Human Rights. Paris:Unesco;2006.

2. Council of Europe. Convention for the Protection of Human Rights and Dignity of the Human Being with regard to the Application of Biology and Medicine: Convention on Human Rights and Biomedicine; 1997.

3. Provides for the National Information Technology Policy and provides other. DOU de 30/10/84, Brazil; 1984.

4. Brazilian Civil Code and related legislation. Brazil; 2008.

5. Resolution No 196 of 10 October. Brazil; 1996.
6. Resolution No 196 of 12 December. Brazil; 2012.

7. Botelho LLR, Cunha CCA, Macedo M. The Integrative Review method in organizational studies. Management and Soc. 2011;5(11):121-136.

8. Lacerda MR, Costenaro RGS. Research methodologies for nursing and health from theory to practice. Porto Alegre, SP: Moriá Editora LTDA; 2015.

9. Means JM, Kodner IJ, Brown D, et al. Sharing clinical photographs: Patient rights, professional ethics, and institutional responsibilities. Int $J$ Clin Pract. 2015;69(4):17-22.

10. Bronsard N, Chignon SB, Amoretti N, et al. Interest of including trauma photography in the picture archiving and communication system of a teaching hospital. Orthop Traumatol Surg Res. 2015;101(3):387-390.

11. Burns K, Belton S. Clinicians and their cameras: policy, ethics and practice in an Australian tertiary hospital. Aust Health Rev. 2013;37(4):437-441.

12. Lima AIC, Cruz RA, Silva RA. Analysis of the profiles of dental clinics and dentists in two social networks regarding ethical, advertising and publicity aspects. RBOL-Rev Bras Odontol Leg. 2016;3(2).

13. Villamizar PJ, Moreno SM, Moreno F, et al. Management of electronic social networks by medical students: the case of the publication of photographs with patients and medical professionalism. Biomedical. 2015;36(1):140-148.

14. Devakumar D, Brotherton H, Halbert J, et al. Taking ethical photos of children for medical and research purposes in low-resource settings: an exploratory qualitative study. BMC Medical Ethics. 2013;14:27.

15. Federal Council of Medicine. Code of Medical Ethics. Resolution CFM $\mathrm{n}^{\mathrm{o}} 1.931$ / 2009. Official Journal of the Union. 2009.

16. Federal Council of Medicine. Official CFM No 8.369 / 2018-DEPCO. Brazil. 2018.

17. Conselho Federal University of Dentistry. Code of Ethical Dentistry. Resolution CFO No 118/2012. Official Journal of the Union. Brazil; 2012 .

18. Federal nursing. Code of Ethics of Nursing. Resolution COFEN No 564/2017. Official Journal of the Union. Brasília, 2017. 